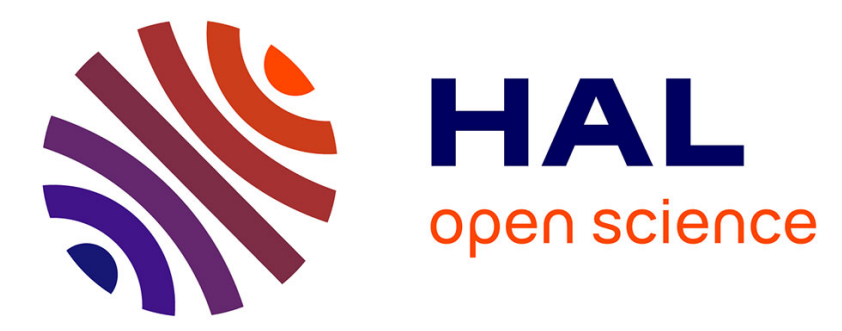

\title{
Interband and intersubband transitions in photoexcited mixed type I and type II GaAs/AlAs superlattices
}

\author{
Y. Garini, E. Linder, E. Cohen, D. Gershoni, E. Ehrenfreund, A. Ron, L.
}

Pfeiffer

\section{- To cite this version:}

Y. Garini, E. Linder, E. Cohen, D. Gershoni, E. Ehrenfreund, et al.. Interband and intersubband transitions in photoexcited mixed type I and type II GaAs/AlAs superlattices. Journal de Physique IV Proceedings, 1993, 03 (C5), pp.C5-241-C5-244. 10.1051/jp4:1993546 . jpa-00251632

\section{HAL Id: jpa-00251632 https://hal.science/jpa-00251632}

Submitted on 1 Jan 1993

HAL is a multi-disciplinary open access archive for the deposit and dissemination of scientific research documents, whether they are published or not. The documents may come from teaching and research institutions in France or abroad, or from public or private research centers.
L'archive ouverte pluridisciplinaire HAL, est destinée au dépôt et à la diffusion de documents scientifiques de niveau recherche, publiés ou non, émanant des établissements d'enseignement et de recherche français ou étrangers, des laboratoires publics ou privés. 


\title{
Interband and intersubband transitions in photoexcited mixed type I and type II GaAs/AlAs superlattices
}

\author{
Y. GARINI, E. LINDER, E. COHEN, D. GERSHONI, E. EHRENFREUND, A. RON and \\ L.N. PFEIFFER*
}

Solid State Institute, Technion-Israel Institute of Technology, Haifa 32000, Israel

* AT\&T Bell Laboratories, Murray Hill, NJ 07974, U.S.A.

\begin{abstract}
We present a study of the interband and intersubband transitions in mixed type I and type II GaAs/AlAs superlattices. Depending on the energy and intensity of the exciting (laser) radiation, either excitons in the wide GaAs wells or separate 2DEG and 2DHG are formed in the wide and narrow GaAs wells, respectively. We observe both the direct interband transitions and the inter-well transitions, and identify them by comparison with the calculated conduction and valence subband dispersion relations and resulting spectra. Intersubband transitions of the photoexcited 2DEG are observed as well. Under the application of an external magnetic field, the interband transitions between electron and hole Landau levels are shown to be strongly dependent on the density of the photoexcited 2DEG.
\end{abstract}

\section{INTRODUCTION}

The energy of the $\Gamma$ and $X$ conduction band minima in GaAs/AlAs superlattices (SL) is strongly dependent on quantum confinement [1]. A SL will have a direct or indirect $\left(e_{1}-h h_{1}\right)$ bandgap, in both real and reciprocal spaces, depending on whether the $\Gamma$ or $X$ minimum is lowest (type I or type II SL, respectively). Galbraith et al [2] recently introduced a mixed type I - type II SL (MTSL) by growing alternating wide $\left(L_{W}>35 \AA\right)$ and narrow $\left(L_{N}<35 \AA\right)$ GaAs layers, separated by AlAs layers. A schematic description of such a MTSL is shown in Fig. 1 by the variation of the conduction and valence band extrema along the growth direction (z-axis).

Galbraith et al [2] studied the absorption spectrum of such a MTSL, as a function of photoexcitation intensity, when the laser energy is tuned above the narrow well bandgap. They showed that a two-dimensional electron gas (2DEG) is formed in the wide well, and the absorption spectrum is strongly modified as its density increases. In this work we studied the interband photoluminescence (PL) and its excitation (PLE) spectra as well as the electron intersubband transitions observed by photoinduced absorption (PIA) in MTSL's. We show that all these transitions are affected by the presence of a photoexcited 2DEG. 

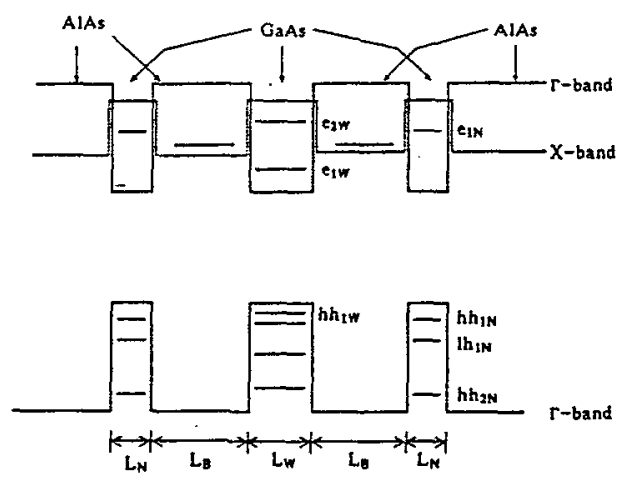

Fig. 1: A schematic description of the band structure of the mixed type I - type II superlattice studied here.

\section{EXPERIMENT}

The MTSL's were grown by molecular beam epitaxy on (001)-oriented GaAs substrates. Several structures with various $L_{W}$ and $L_{N}$ (cf. Fig. I) were studied, but we report here only on the results obtained with $L_{W}=68 \AA$ (24 monolayers), $L_{N}=26 \AA$ (9 ml's) and $L_{B}=102 \AA$ (36 ml's). The structure is symmetric, namely, every wide well has a neighboring narrow well on each side. For interband spectroscopy, the samples were placed in an immersion-type dewar ( $\mathrm{T}=2 \mathrm{~K}$ ). A magnetic field ( $B \leq 5$ Tesla) could be applied parallel to the z-axis. The samples were excited by $A r^{+}$and dye lasers and the luminescence was measured with a double monochromator. For the intersubband spectroscopy, the samples were cut and polished at $45^{\circ}$ to the z-axis. In this 'waveguide configuration' the infrared radiation had an electric field component along the z-axis. In these experiments, the samples were placed in an immersion type dewar, with an ambient temperature in the range of 2-100K. The spectra were obtained using a Fourier transform infrared spectrometer.

Fig. 2 shows the interband PL transitions of the MTSL obtained under excitation with laser energy of $E_{L}=1.65 \mathrm{eV}$, below the $\left(e_{1}: h h_{1}\right)_{N}$ bandgap of the narrow well (Fig. $2 \mathrm{a}$ ) and with $E_{L}=2.41$ $\mathrm{eV}$, above this bandgap (Fig. 2b). Fig. 2c shows the PLE, monitored at the strongest transition, $\left(e_{1}: h h_{1}\right)_{w}$. Fig. 3 shows the PIA (intersubband transitions) under similar excitation conditions as those used in Figs. 2a,b (but observed at various ambient temperatures). Finally, Fig. 4 shows the effects of an applied magnetic field on the PLE spectrum (of the $\left(e_{1}: h h_{1}\right)_{W}$ band) in the spectral range of the $\left(e_{1 W}-h h_{1 W}\right)$ and $\left(e_{1 W}-l h_{1 W}\right)$ transitions.

\section{DISCUSSION}

The PLE spectrum (Fig. 2c) shows several wide well transitions and the lowest $\left(e_{1}: h h_{1}\right)_{N}$ transition of the narrow well. We have calculated the valence and conduction subband dispersion curves using the method of Baraff and Gershoni [3]. The arrows shown in this figure indicate the calculated interband transitions (exciton binding energy and inhomogeneous broadening are disregarded). The PL spectra show two types of transitions. In Fig. 2a the $\left(e_{1}: h h_{1}\right)_{W}$ band is observed. This is the excitonic transition of the wide wells. When the excitation is above the $\left(e_{1 N}-h h_{1 N}\right)$, (Fig. $2 \mathrm{~b})$, the PL spectrum still shows the $\left(e_{1 W}-h h_{1 W}\right)$ e-h pair recombination and it is the strongest feature. However, much weaker bands (denoted W-N) are observed in the range of 1.7-1.8 eV. The relative intensity of these bands depends on the excitation intensity. We attribute them to the radiative recombination of electrons in $e_{1 w}$ (the $2 \mathrm{DEG}$ of the wide wells) with holes in $h h_{1 N}$ and $l h_{1 N}$ (the $2 \mathrm{DHG}$ of the narrow wells). These are indirect transitions in real space (but direct in reciprocal space). These separately confined $e$ and $h$ gases are formed because the tunneling time of the electrons from the narrow to the wide wells [2]. This process is governed by the fast $\Gamma-X$ scattering time in the AlAs barriers [4]. The hole tunneling time, on the other hand, is much slower, as we verified by measuring the PL time dependence under short, pulsed excitation. 


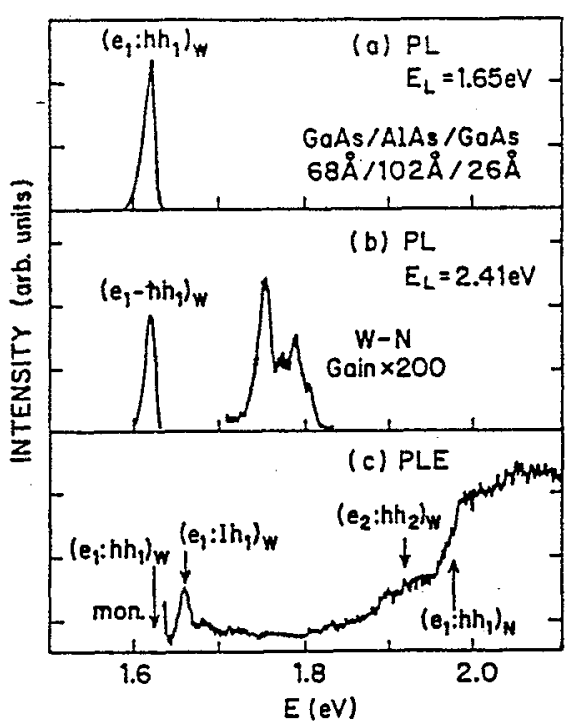

Fig. 2: The interband transitions observed by photoluminescence excited: (a) below the narrow well lowest bandgap $\left(E_{L}<\left(e_{1 N}-h h_{1 N}\right)\right.$. (b) above it. (c) The photoluminescence excitation spectrum.

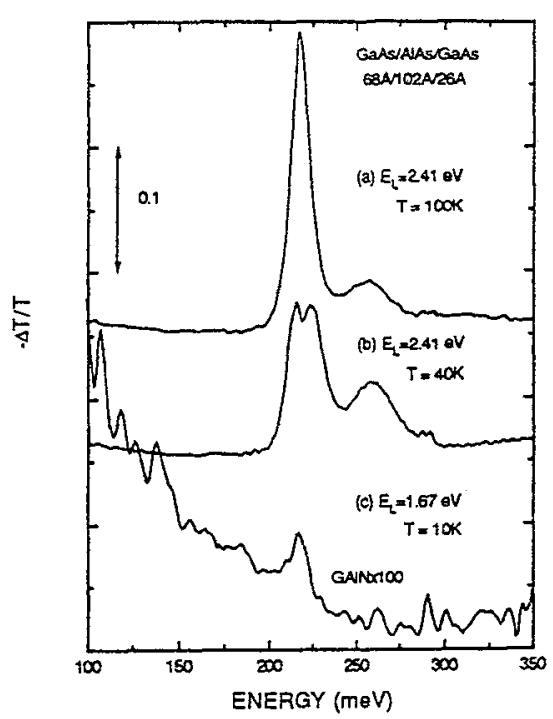

Fig. 3: The photoinduced absorption spectra in the spectral range of the $\left(e_{1 W}-e_{2 W}\right)$ transition. In (a) and (b) $E_{L}>\left(e_{1 N}-h h_{1 N}\right)$ and the 2DEG in the wide well gives rise to the observed transitions. In (c) $E_{L}<\left(e_{1 N}-h h_{1 N}\right)$ and the transition is due to $\left(e_{1}: h h_{1}\right) w$ excitons.

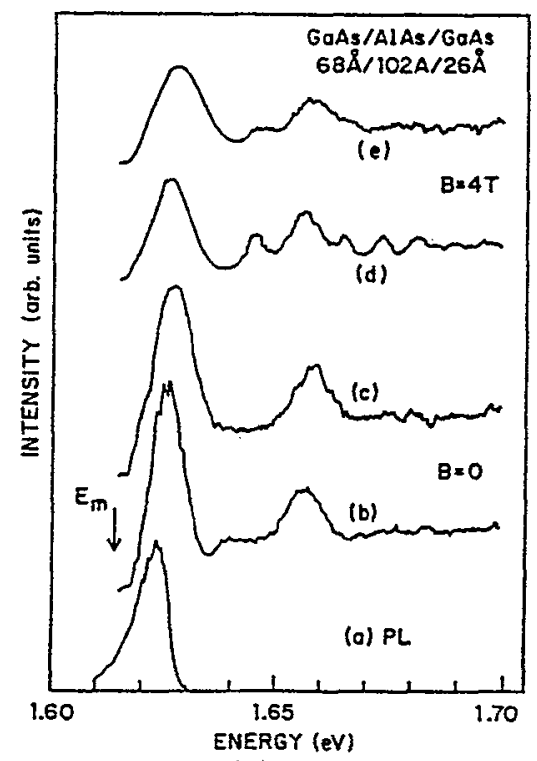

Fig. 4: (a) The $\left(e_{1}: h h_{1}\right)_{W}$ PL spectrum. (b) The PLE spectrum monitored at the $\left(e_{1}: h h_{1}\right)_{W}$ PL. (c) Same as (b) but with an additional photoexcitation with $E_{L}>\left(e_{1 N}-h h_{1 N}\right)$, so that a 2DEG is formed in the wide well. (d) and (e) are similar to (b) and (c) but under a magnetic field applied along the growth direction. 
The intersubband PIA spectrum (Figs. 3a,b) consists of a strong band (at $220 \mathrm{meV}$ ) which corresponds to the $\left(e_{1 W}-e_{2 W}\right)$ transition of the 2DEG in the wide wells. (This identification is supported by the calculated conduction subband energies). For an excitation energy $E_{L}>\left(e_{1 N}-h h_{1 N}\right)$, this transition is strong and long-lived. However, for $E_{L}<\left(e_{1 N}-h h_{1 N}\right)$, the $\left(e_{1 W}-e_{2 W}\right)$ transition shown in Fig. 3c, is due to very short-lived excitons in the wide well. It is much weaker than in the case of the 2DEG because the steady state density of the exciton population is much smaller than that of the 2DEG. The other band observed by PIA, centered at $260 \mathrm{meV}$, has a different dependence on temperature and on excitation intensity than the $\left(e_{1 W}-e_{2 W}\right)$ transition. The calculated dispersion curves show that there are no other electronic intersubband transitions in this spectral range. However, a combined $\left(e_{1} w-e_{2} w\right)$ and $\left(h h_{1} w-l h_{1 W}\right)$ transitions may give rise to the broad band centered at $260 \mathrm{meV}$. For such a cooperative transition to occur, there must be a free hole population in the wide wells. We have recently shown [5], that long-lived holes can be photoexcited in the presence of a 2DEG, provided that the electron density is smaller than $7 \times 10^{10} \mathrm{~cm}^{-2}$. In the present case of the MTSL's, holes are present in the wide wells and their interaction with the 2DEG may give rise to the cooperative transition.

Finally, we consider the effect of the 2DEG on the interband transitions between the $e_{1}$ and $h h_{1}$ Landau levels. Figs. $4 \mathrm{~b}, \mathrm{c}$ show the PLE spectra monitored in the $\left(e_{1}-h h_{1}\right)$ emission. The spectrum in Fig. $4 \mathrm{c}$ differs from that of Fig. $4 \mathrm{~b}$ in that it is simultaneously excited with an $E_{L}=2.41 \mathrm{eV}$ laser, so that a $2 D E G$ is formed. The spectra of Figs. $4 \mathrm{~d}, \mathrm{e}$ taken under similar conditions as in Figs. $4 b, c$, but with an applied magnetic field of $B=4 T$, are shown in Figs. 4d,e. The well known [6] Landau level splitting is observed when there is no 2DEG present. However, these levels are virtually washed out when there is an additional photoexcitation with $E_{L}=2.41 \mathrm{eV}$ generating the 2DEG. This means that e-e and e-h scattering in the 2DEG broadens up the Landau levels to the extent that they cannot be observed.

In summary, we showed that the presence of a photoexcited 2DEG has profound effects on the interband and intersubband transitions in the mixed type I - type II SL's. While we can account for some of the observed transitions by comparing their energies with the calculated ones, many problems are still open and under current study.

Acknowledgments The work at the Technion was supported by the US- Israel Binational Science Foundation (BSF), Jerusalem, Israel, and by the Basic Research Foundation Administered by the Israel Academy of Sciences and Bumanities, and was carried out in the Center for Advanced OptoElectronics.

\section{References}

[1] Cingolani R., Ploog K., Scamarcio G. and Tapfer L., Opt. and Quan. Elect. 22 (1990) S201-S230.

[2] Galbraith I., Dawson P. and Foxon C.T., Phys. Rev. B45 (1992) 13499-13508.

[3] Baraff G.A. and Gershoni D., Phys. Rev. B43 (1991) 4011-4022.

[4] Feldman J., Nunnenkamp J., Peter G., Göbel E., Kuhl J., Ploog K., Dawson P. and Foxon C.T., Phys. Rev. B42 (1990) 5809-5821.

[5] Garini Y., Ehrenfreund E., Cohen E., Ron Arza, Law K-K., Merz J.L. and Gossard A.C., Phys. Rev B48 (in press, 1993).

[6] Heimañ D., "The Spectroscopy of Semiconductors", Edited by D.J. Seiller and C.L. Litter, (Academic, New York 1992), Vol. 36, pp. 16-48. 